\title{
Cloning and characterization of the SERK1 gene in triploid Pingyi Tiancha [Malus hupehensis (Pamp.) Rehd. var. pingyiensis Jiang] and a tetraploid hybrid strain
}

\author{
L.J. Zhang ${ }^{1,3}$, W.X. Dong ${ }^{1}$, S.M. Guo', Y.X. Wang ${ }^{2}$, A.D. Wang ${ }^{1}$ and X.J. Lu ${ }^{3}$ \\ ${ }^{1}$ College of Horticulture, Shenyang Agricultural University, Shenyang, Liaoning, China \\ ${ }^{2}$ Yantai Agricultural Science and Technology Institute, Yantai, Shandong, China \\ ${ }^{3}$ College of Forestry, Shenyang Agricultural University, Shenyang, Liaoning, China \\ Corresponding author: W.X. Dong \\ E-mail: wxdong63@126.com
}

Genet. Mol. Res. 14 (4): 14576-14586 (2015)

Received July 20, 2015

Accepted September 18, 2015

Published November 18, 2015

DOI http://dx.doi.org/10.4238/2015.November.18.21

\begin{abstract}
This study aims to explore the roles of somatic embryogenesis receptor-like kinase (SERK) in Malus hupehensis (Pingyi Tiancha). The fulllength sequences of SERK1 in triploid Pingyi Tiancha (3n) and a tetraploid hybrid strain 33\# (4n) were cloned, sequenced, and designated as MhSERK1 and MhdSERK1, respectively. Multiple alignments of amino acid sequences were conducted to identify similarity between MhSERK1 and MhdSERK1 and SERK sequences in other species, and a neighbor-joining phylogenetic tree was constructed to elucidate their phylogenetic relations. Expression levels of MhSERK1 and MhdSERK1 in different tissues and developmental stages were investigated using quantitative real-time PCR. The coding sequence lengths of MhSERK1 and MhdSERK1 were $1899 \mathrm{bp}$ (encoding 632 amino acids) and 1881 bp (encoding 626 amino acids), respectively. Sequence analysis demonstrated that MhSERK1 and MhdSERK1 display high similarity to SERKs in other species, with a conserved intron/exon structure that is unique to members of the SERK
\end{abstract}


family. Additionally, the phylogenetic tree showed that MhSERK1 and MhdSERK1 clustered with orange CitSERK (93\%). Furthermore, MhSERK1 and MhdSERK1 were mainly expressed in the reproductive organs, in particular the ovary. Their expression levels were highest in young flowers and they differed among different tissues and organs. Our results suggest that MhSERK1 and MhdSERK1 are related to plant reproduction, and that $M h S E R K 1$ is related to apomixis in triploid Pingyi Tiancha.

Key words: Malus; Apomixis; Somatic embryogenesis receptor-like kinase; Expression analysis

\section{INTRODUCTION}

Apomixis is a special type of asexual reproduction, where plants form embryos and produce viable offspring, which are genetically identical to the mother plant, via seeds without sperm-egg fusion (Rodriguez-Leal and Vielle-Calzada, 2012). Apomixis is a special reproductive mode of asexual reproduction found among higher plants, which simplifies the process of cross breeding and leads to further stable hybrid vigor in plant breeding (Spillane et al., 2001). Therefore, apomixis may help plants to retain successful genotypes and propagate specific genotypes or superior hybrids bearing complex gene sets, resulting in a fitness advantage in the surrounding environment (Albertini et al., 2005; Sailer et al., 2014).

Receptor-like kinases (RLKs) are pivotal components in the regulation of hormone signaling, abiotic and biotic stress responses, and development in plants (Wrzaczek et al., 2010), and the leucine-rich repeat sequence receptor-like kinase (LRR-RLK) is a large subfamily of the RLK family in plants. Somatic embryogenesis receptor-like kinase (SERK) was first discovered in embryogenic cells in the hypocotyl of Daucus carota, and was shown to play an important role in plant life processes (Schmidt et al., 1997). LRR-RLKs are divided into 13 subclasses according to the structure and quantity of different extracellular LRRs (Gou et al., 2010) 2010, and SERK belongs to the second subclass (Shiu and Bleecker, 2001). The protein structure of SERK begins with a signal N-terminal peptide followed by one leu-zipper domain, five LRRs, one ser-pro-ro (SPP) motif, one single transmembrane (TM) domain, three conserved serine/threonine kinase subdomains, and one C-terminal leucine-rich domain (Cueva et al., 2012).

Several studies have shown that SERK is involved in apomixis (Shi et al., 2012). Albertini et al. discovered that PpSERK acts as the switch that channels the development of the embryo sac, and it may also redirect signaling gene products to compartments other than their typical ones in nucellar cells of apomictic genotypes (Albertini et al., 2005). Podio et al. demonstrated that the expression of PnSERK2 in nucellar tissue is correlated with apomixis onset in Paspalum notatum (Podio et al., 2014). At present, several SERKs have been cloned in both dicotyledonous and monocotyledonous plants, such as Arabidopsis thaliana (Hecht et al., 2001), Medicago truncatula (Nolan et al., 2003), Helianthus annuus (Thomas et al., 2004), Oryza sativa (Hu et al., 2005; Ito et al., 2005), Rosa hybrida cv. Linda (Zakizadeh et al., 2010), Triticum aestivum (Singla et al., 2007), Vitis vinifera (Schellenbaum et al., 2008), and Zea mays (Baudino et al., 2001). However, reports of SERKs in triploid Pingyi Tiancha (Malus hupehensis Pamp. Rehd. var. pingyiensis Jiang) and a tetraploid hybrid strain 33\# have not been published. In addition, a previous report has also proven that SERK functions are highly conserved (Shi et al., 2012). Thus, SERKs should exist in triploid Pingyi Tiancha and the tetraploid hybrid strain 33\#. 
The apomictic plant Pingyi Tiancha, which originates from Pingyi county in the Shandong province of China, shows favorable characteristics, such as salt tolerance (Dai et al., 2011), high grafting affinity with apple, and uniform seedlings (Liu et al., 1989). Apomixis is well known for its ability to fix these advantageous genotypes (Sailer et al., 2014). Therefore, it is of high importance to explore the mechanism of apomixis in Pingyi Tiancha.

In this study, to determine the role of SERK in M. hupehensis development and reproduction, SERK was cloned from two of these plants, triploid Pingyi Tiancha and a tetraploid hybrid strain 33\#. In addition, protein structures and phylogenetic relations of the cloned SERKs were analyzed. Furthermore, quantitative real-time PCR (qRT-PCR) was used to analyze the expression levels of $S E R K$ in different tissues, organs, and ovaries during different developmental stages.

\section{MATERIAL AND METHODS}

\section{Plant material}

Triploid Pingyi Tiancha (3n) and the tetraploid hybrid strain 33\# (4n; obtained from the cross of Pingyi Tiancha $x$ Zha'ai Shandingzi) were cultivated in the experimental farm at Shenyang Agricultural University. Young leaves were used for DNA and RNA extraction. RNA from leaves, sepals, petals, ovaries, pistil, and stamen was used for gene expression analysis. All specimens were immediately frozen in liquid nitrogen after collection, and then stored at $-70^{\circ} \mathrm{C}$.

\section{RNA/DNA extraction and cDNA synthesis}

Total RNA was extracted using the conventional cetyltrimethylammonium bromide (CTAB) method (Khan et al., 2004), and poly(A) RNA was isolated using the mRNA Purification Kit (Takara Bio Inc., Otsu, Shiga, Japan) following the manufacturer protocol. RNA preparations were then treated with Dnase I (Takara) and subsequently reversely transcribed into cDNA using AMV reverse transcriptase (Takara). Parallel amplification reactions from $18 \mathrm{~S}$ rRNA were performed as a control. Genomic DNA was extracted from young leaves using the CTAB method (Khan et al., 2004) and samples were treated with Rnase A (Takara) to eliminate RNA.

\section{Cloning the full-length cDNA of SERK1}

A specific gene primer pair SERK1-F1/R1 was designed based on the conserved regions of SERK1 for fragment PCR. Subsequently, rapid amplification was performed for the full-length cDNA sequence of SERK1 utilizing the specific primer pair SERK1-F2/R2. PCR conditions were as follow: initial denaturation at $94^{\circ} \mathrm{C}$ for $5 \mathrm{~min}$, followed by 35 cycles of denaturation at $94^{\circ} \mathrm{C}$ for $40 \mathrm{~s}$, annealing at $60^{\circ} \mathrm{C}$ for $1 \mathrm{~min}$, extension at $72^{\circ} \mathrm{C}$ for $1.5 \mathrm{~min}$, and a final extension at $72^{\circ} \mathrm{C}$ for $10 \mathrm{~min}$. Sequences were sequenced, aligned, and analyzed using DNAMAN (Lynnan Corp, Pointe-Claire, Quebec, Canada) and the Clustal software tools (http://www.ebi.ac.uk/tools/clustalw2) (Larkin et al., 2007). All the primers are listed in Table 1.

\section{Protein structure and phylogenetic analysis for SERK1}

The basic local alignment search tool (BLAST) from the National Center for Biotechnology Information (NCBI) website (http://blast.ncbi.nlm.nih.gov/Blast.cgi) was used to retrieve the results 
of sequencing, and DNAMAN6.0 (Lynnan Corp, Pointe-Claire, Quebec, Canada) was used to analyze sequences. The open reading frame (ORF) for SERK1 was predicted using the ORF Finder tool (http://www.ncbi.nlm.nih.gov/gorf/gorf.html), and then ORFs were translated into amino acid sequences. The physico-chemical parameters of the protein structures were obtained using ExPASy (http://expasy.org/tools/protaram.html/). The phylogenetic relationship of SERK1 in Pingyi Tiancha and the hybrid strain (33\#) was assessed with an additional 17 SERKs from other species using a neighbor-joining tree in ClustalX (Thompson et al., 1994)1994 and MEGA4 (http://www.megasoftware.net/mega4/mega.html) (Tamura et al., 2007) with 1000 replications.

Table 1. Primers used for the amplification and expression analysis of SERK1 in two types of Malus hupehensis, triploid Pingyi Tiancha and tetraploid hybrid strain 33\#.

\begin{tabular}{lll}
\hline Primer & Sequence $\left(5^{\prime}-3^{\prime}\right)$ & Use \\
\hline SERK1-F1 & GAAGTTCATCTTGGGCAGC & Isolation of SERK1 fragments \\
SERK1-R1 & CCCACAACAGCCTCAAAC & Isolation of SERK1 fragments \\
SERK1-F2 & GGATGGAGAGAAAGTTGGGAAT & Amplification of full-length CDS of SERK1 \\
SERK1-R2 & ACTTACCTTGGACCGGATAACTC & Amplification of full-length CDS of SERK1 \\
SERK1-F3 & CGGTTACTTGTTTATCCTT & Real time RT-PCR of SERK1 \\
SERK1-R3 & GGTGAATAATCTTCGGGTC & Real time RT-PCR of SERK1 \\
18S-F & GTAGTCATATGCTTGTCT & Real time RT-PCR of 18S rRNA \\
$18 S-R$ & GAATGATGCGTCGCCAGCACAAAGG & Real time RT-PCR of 18S rRNA \\
\hline
\end{tabular}

\section{qTR-PCR analysis of SERK1}

The primer pair SERK1-F3/R3, which spans two exons, was designed for qRTPCR (Table 1). qRT-PCR was performed using SuperReal PreMix SYBR Green (TIANGEN BIOTECH, Beijing, China) on an iQ5 real-time PCR System (Biorad Laboratories Inc., Hercules, California, USA). Parallel amplification for 18S rRNA was also performed as the endogenous control. Dnase I (Takara) was used to eliminate genomic DNA contamination. Amplification specificity was verified by electrophoresis and melt curve analysis. The qRT-PCR reaction procedure was as follows: $95^{\circ} \mathrm{C}$ for $3 \mathrm{~min}$; followed by 40 cycles of denaturation at $95^{\circ} \mathrm{C}$ for 10 $\mathrm{s}$, annealing at $60^{\circ} \mathrm{C}$ for $20 \mathrm{~s}$, and extension at $72^{\circ} \mathrm{C}$ for $20 \mathrm{~s}$. Each experiment was performed in triplicate. The results were analyzed using the iQ5 System software according to the $2^{-\Delta \Delta C t}$ method (Livak and Schmittgen, 2001).

\section{RESULTS}

\section{Cloning of the SERK1 gene in Malus}

Using the gene primer pair SERK1-F1/R1, we obtained two fragments $705 \mathrm{bp}$ in length from Pingyi Tiancha and the hybrid strain (33\#), respectively. BLAST searches based on these two sequences against the apple genome databases (http://www.applegene.org/and http://gfdb.sdau. edu.cn/) revealed several hits matching SERK conserved domains. Thus, these two fragments were named MhSERK1 and MhdSERK1 for Pingyi Tiancha and the hybrid strain (33\#), respectively. The full-length cDNA sequences of MhSERK1 and MhdSERK1, amplified using primer pair SERK1-F2/R2, were 1899 bp (GenBank accession No. JQ231272) and 1881 bp (GenBank accession No. JQ231273), respectively. CDNA sequences of MhSERK1 and MhdSERK1 contained 11 exons interspersed by 10 introns. The cDNA sequence similarity between triploid and tetraploid $M$. hupehensis was $95.95 \%$, 
with only 18 bp differences (696 to 714 bp; Figure 1). The cDNA sequences had an integral ORF with the initial codon ATG at the 5'-end and the terminal codon TAA at the 3'-end.
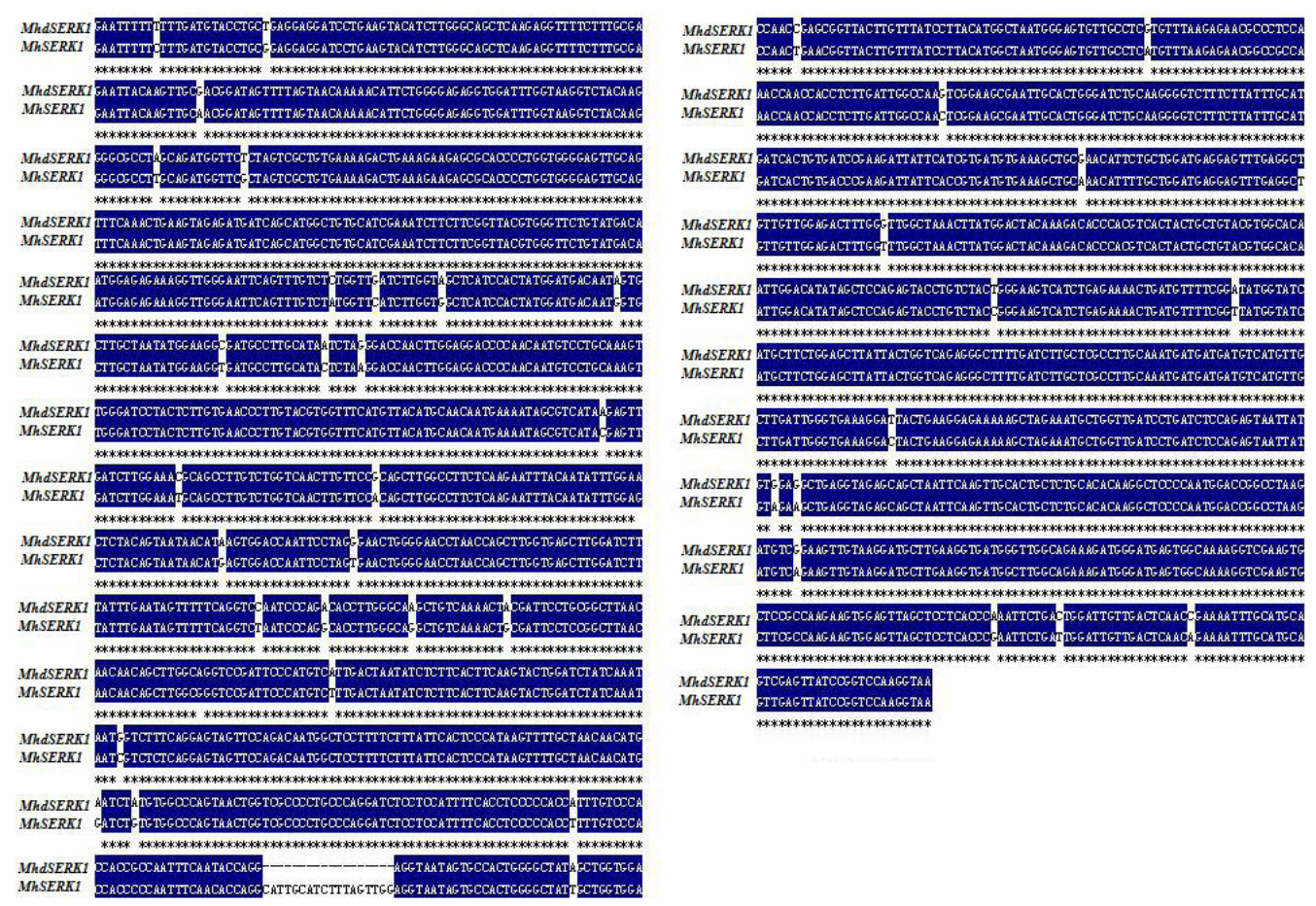

Figure 1. Full-length cDNA sequence alignment of SERK1 in two types of Malus hupehensis, triploid Pingyi Tiancha (MhSERK1) and tetraploid hybrid strain 33\# (MhdSERK1).

\section{Coding sequence characteristics and clustering analysis of MhSERK1 and MhdSERK1}

MhSERK1 and MhdSERK1 encoded peptides of 632 and 626 amino acids with the predicted molecular weights of 69.58 and $68.86 \mathrm{KDa}$ and isoelectric points (PI) of 5.22 and 5.45, respectively. MhSERK1 and MhdSERK1 possessed 11 domains, including a signal peptide, followed by one leucine zipper domain, four LRR domains with glycosylation sites, one proline-rich domain containing an SPP motif, one alanine-rich hydrophobic TM domain, and three serine/threonine kinase domains. Moreover, MhSERK1 and MhdSERK1 had one extracellular signal domain, one standard TM domain, and one intracellular kinase domain. Multiple alignments of amino acid sequences of MhSERK1 and MhdSERK1 with other SERK members are shown in Figure 2. The phylogenetic tree revealed that MhSERK1 and MhdSERK1 clustered into the same subfamily with orange CitSERK (Figure 3). Additionally, MhSERK1 and MhdSERK1 showed high similarities to CitSERK1 (SERK1 from Citrus unshiu, 93\%), PtSERK1 (SERK1 from Populus tomentosa, 91\%), AtSERK2 (SERK2 from Arabidopsis thaliana, 90\%), ZmSERK2 (SERK2 from Zea mays, 85\%), ZmSERK1 (SERK1 from Zea mays, 84\%), and AtSERK3 (SERK3 from Arabidopsis thaliana, 77\%). 


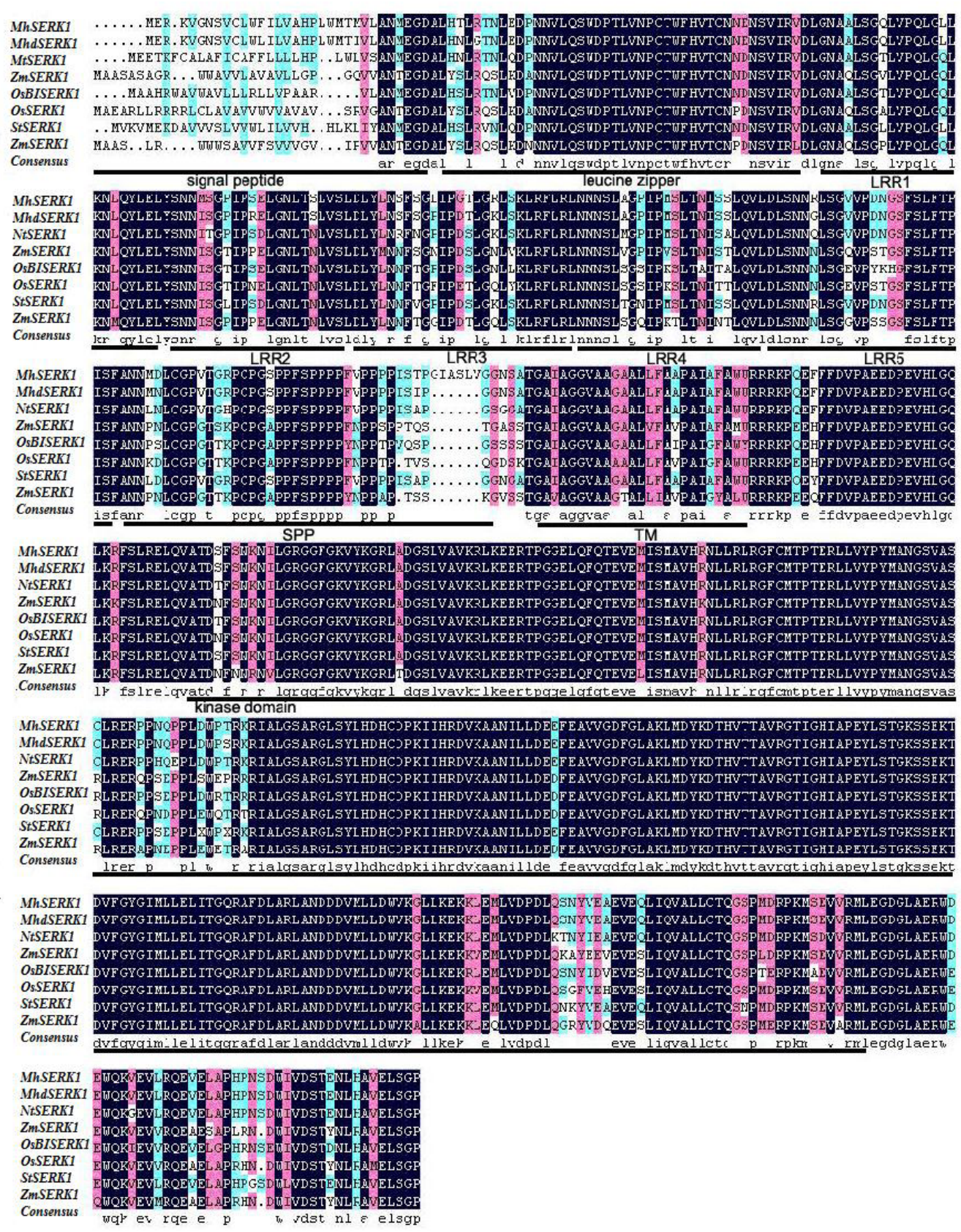

Figure 2. Amino acid sequence alignment of SERK1 in two types of Malus hupehensis, triploid Pingyi Tiancha (MhSERK1) and tetraploid hybrid strain 33\# (MhdSERK1). Consensus are shaded in black; relevant conserved domains are indicated below the alignment; gaps $\left(^{*}\right)$ were included in the sequences to maximize the alignment; LRR1LRR5, leucine-rich repeats; SPP, proline rich region; TM, transmembrane domain; CitSERK is from orange sequences; PtSERK1 is from Populus tomentosa; AtSERK2 and AtSERK3 are from the Arabidopsis thaliana; ZmSERK1 and ZmSERK2 are from Zea mays. 


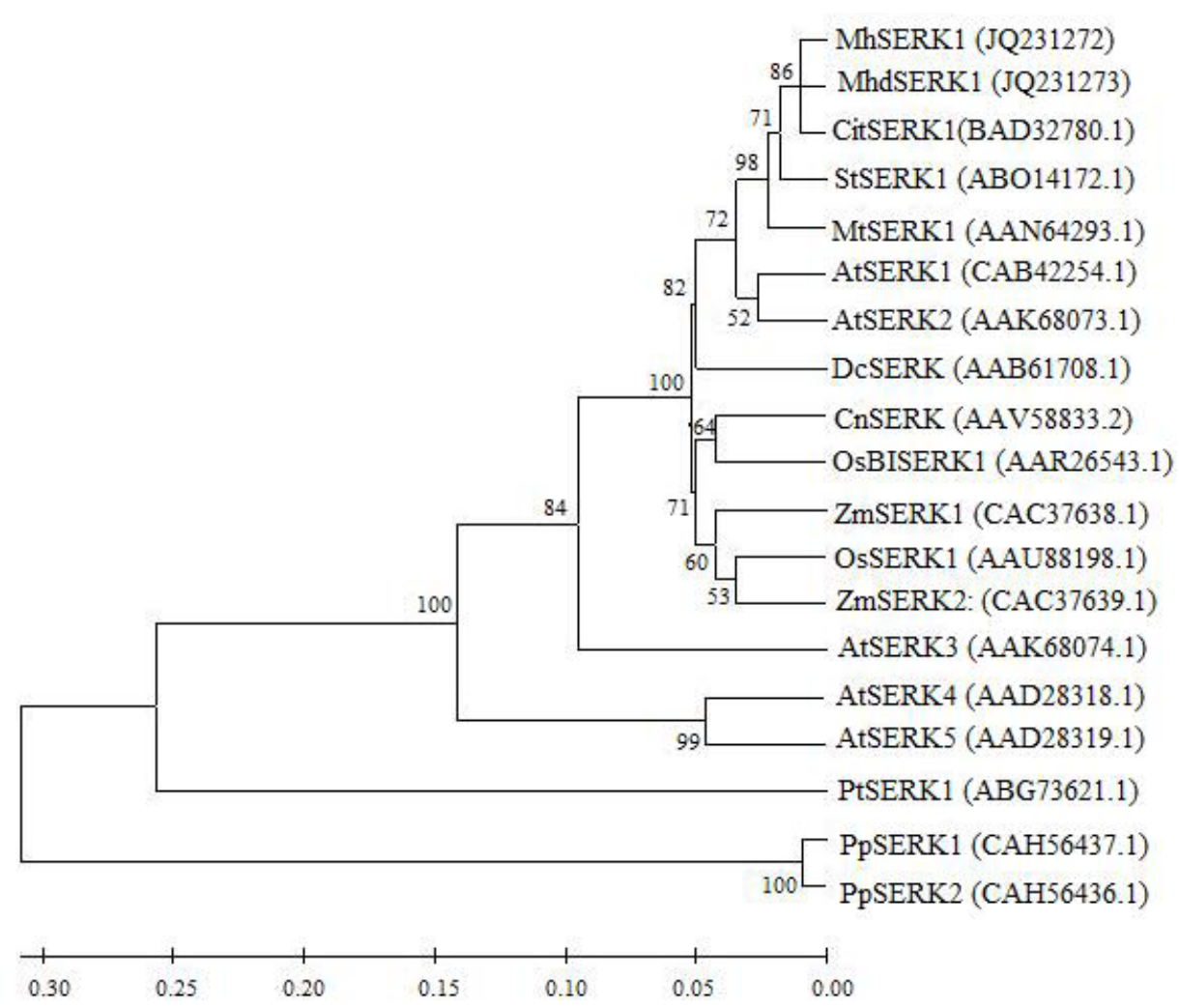

Figure 3. Cluster analysis of SERK1 from two types of Malus hupehensis, triploid Pingyi Tiancha (MhSERK1) and tetraploid hybrid strain 33\# (MhdSERK1) with other SERKs using amino acid sequences. The numbers along the tree branches refer to GenBank accession Nos. of SERKs. CitSERK1, SERK1 from Citrus unshiu; StSERK1, SERK1 from Solanum tuberosum; MtSERK1, SERK1 from Medicago truncatula; AtSERK1, SERK1 from Arabidopsis thaliana; AtSERK2, SERK2 from Arabidopsis thaliana; AtSERK3, SERK3 from Arabidopsis thaliana; AtSERK4, SERK4 from Arabidopsis thaliana; AtSERK5, SERK5 from Arabidopsis thaliana; DcSERK, SERK from Daucus carota; CnSERK, SERK from Cocos nucifera; OsBISERK1, SERK1 from Oryza sativa Indica Group; ZmSERK1, SERK1 from Zea mays; ZmSERK2, SERK2 from Zea mays; OsSERK1, SERK1 from Oryza sativa Japonica Group; PtSERK1, SERK1 from Populus tomentosa; PpSERK1, SERK1 from Poa pratensis; PpSERK2, SERK2 from Poa pratensis.

\section{MhSERK1 and MhdSERK1 expression analysis}

qRT-PCR results showed that MhSERK1 gene expression primarily occurred during reproductive growth and remained high throughout the floral organ, with a peak in the ovary of Pingyi Tiancha. For the hybrid strain (33\#), MhdSERK1 was mainly expressed in the stamen and ovary, with a peak in the ovary (Figure 4). Moreover, the expression levels of MhSERK1 in the stamen, pistil, and ovary of Pingyi Tiancha were higher than those in the stamen, pistil, and ovary of the hybrid strain (33\#). Further examination of MhSERK1 and MhdSERK1 expression levels in the ovary of Pingyi Tiancha and the hybrid strain (33\#) during floral development showed that their expression levels were highest during the young flower period, followed by the mature flower period and late anthesis (Figure 5). 


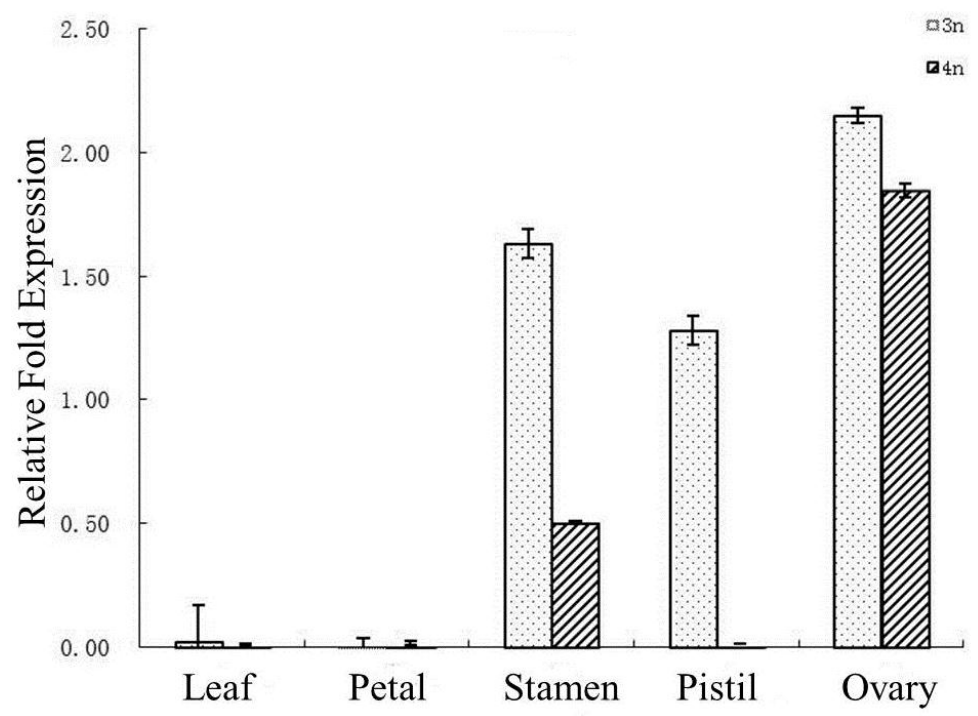

Figure 4. Expression levels of SERK1 in different tissues and organs of two types of Malus hupehensis, triploid Pingyi Tiancha and tetraploid hybrid strain $33 \#$.

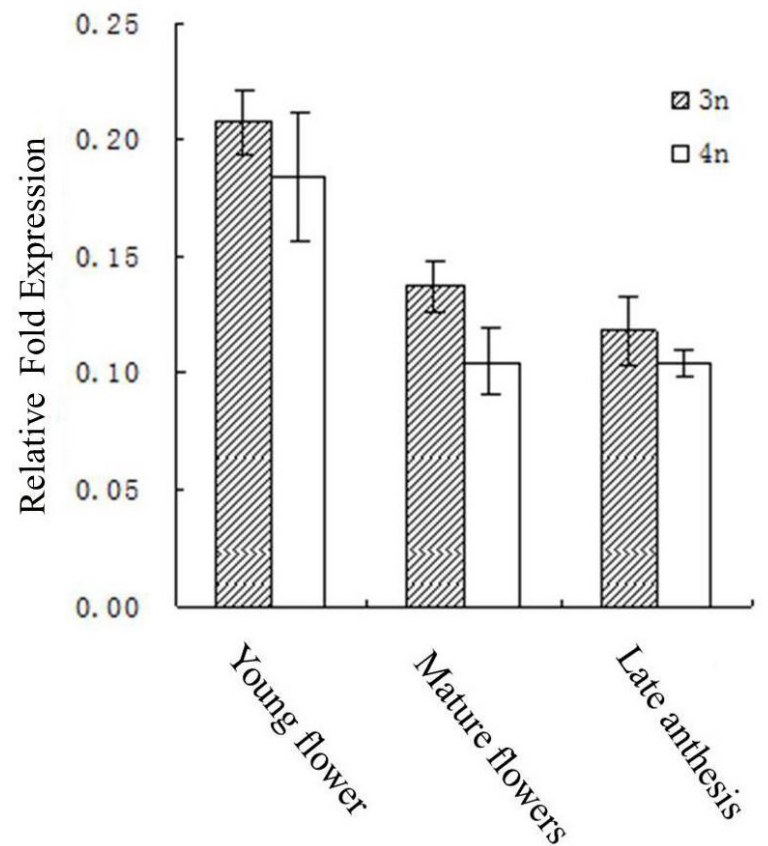

Figure 5. Expression levels of SERK1 in the ovary during different developmental stages (young flowers, mature flowers, late anthesis) in triploid Pingyi Tiancha and tetraploid hybrid strain 33\#. 


\section{DISCUSSION}

In the present study, we cloned the homologous genes of MhSERK1 and MhdSERK1 in triploid Pingyi Tiancha and a tetraploid hybrid strain (33\#), and analyzed their sequence characteristics as well as their expression patterns. The structures of MhSERK1 and MhdSERK1 contained an extracellular binding domain, a TM domain, and an intracellular kinase domain structure, which belongs to the second major category of the LRR-RLK family (Walker, 1994). The structural characteristics of the SERK family were all displayed in MhSERK1 and MhdSERK1, including a signal peptide, one leucine zipper domain, four LRR domains containing glycosylation sites, one proline-rich domain containing one SPP motif, one alanine-rich hydrophobic TM domain, and three serine/threonine kinase domains (Cueva et al., 2012). Additionally, we also discovered that highly conserved exons and exon/intron structure exists in MhSERK1 and MhdSERK1, which is in accordance with the SERK family, indicating the functional significance of MhSERK1 and MhdSERK1 (Sharma et al., 2008).

The phylogenetic analysis revealed that MhSERK1 and MhdSERK1 cluster with CitSERK, which is involved in somatic embryogenesis and plant growth (Ge et al., 2010). MhSERK1 and MhdSERK1 also showed high sequence similarities with other SERK members, such as CitSERK, PtSERK1, AtSERK2, ZmSERK2, ZmSERK1, and AtSERK3. These results suggest that MhSERK1 and MhdSERK1 belong to the SERK family and have similar biological functions.

Studies have demonstrated that SERKs play multiple roles in growth, development, and apomixis in plants (Hecht et al., 2001; Shi et al., 2012). In our research, we discovered that MhSERK1 and MhdSERK1 were highly expressed in the ovary of Pingyi Tiancha and the hybrid strain (33\#), respectively. Former studies have indicated that expression of SERKs in carrot, Dactylis glomerata, and Arabidopsis acts as a biomarker in embryonic cells (Schmidt et al., 1997; Somleva et al., 2000; Hecht et al., 2001). Moreover, SERK1 was involved in the microspore development of plant reproduction and was mainly expressed in the reproductive organs (Albrecht et al., 2005; Colcombet et al., 2005; Shi et al., 2014). In Arabidopsis, the expression level of AtSERK1 mRNA was highest in closed flower buds (Schmidt et al., 1997). Additionally, Podio et al. - revealed that expression of PnSERK2 in nucellar tissue in $P$. notatum is correlated with apomixis onset in the plant (Podio et al., 2014). In this study, we demonstrated that MhSERK1 and MhdSERK1 were highly expressed in the ovary of Pingyi Tiancha and the hybrid strain (33\#) during the young flower period, followed by the mature flower and late anthesis periods, during floral development. All these results suggest that expression of MhSERK1 and MhdSERK1 is related to plant reproduction.

Additionally expression of MhSERK1 in Pingyi Tiancha was found to be higher than expression of MhdSERK1 in the hybrid strain (33\#). Albertini et al. (2005) demonstrated that expression of PpSERK was different in apomictic and sexual genotypes in Poa pratensis1, and thus, PpSERK may be related to apomixis. In addition, Shi et al. have shown that once SERK is activated by the nucellar cell near the megasporocyte of amixis, the migrule may induce signal conduction from nucellar cells to the embryo sac development, and the functional somatic embryo produced by meiosis of somatic cells in the ovary could lead to apomixis (Shi et al., 2012). Accordingly, we speculated that, in our study, MhSERK1 and MhdSERK1 might be related to reproduction in Pingyi Tiancha and the hybrid strain (33\#), whereas MhSERK1 might play a key role in apomixis in Pingyi Tiancha.

Further, the expression levels of MhSERK1 and MhdSERK1 were variable in leaf, petal, pistil, stamen, and ovary tissue. This is consistent with previous research that demonstrated that OsSERK1 showed variable expression levels in different tissues, as well as by the host defense 
against fungal infection and positively regulated somatic embryogenesis of cultured cells (Hu et al., 2005). Moreover, MaSERK1 was found to be associated with disease resistance response and somatic embryogenic competence (Huang et al., 2010). However, further analysis of the functions of MhSERK1 and MhdSERK1 in different organs and tissues is required.

In conclusion, our study demonstrated the role of MhSERK1 and MhdSERK1 in plant development in $M$. hupehensis. High expression of MhSERK1 and MhdSERK1 in ovary tissue indicates their role in plant reproduction and MhSERK1 may be related to apomixis in Pingyi Tiancha. However, further analysis is still required to validate their roles in M. hupehensis development.

\section{Conflicts of interest}

The authors declare no conflict of interest.

\section{ACKNOWLEDGMENTS}

Research supported by the National Natural Science Foundation of China (\#30971975) (Study on the reproductive characteristics and generative mechanism of the tetraploid leaf-wrinkled and dwarf strains in Malus).

\section{REFERENCES}

Albertini E, Marconi G, Reale L, Barcaccia G, et al. (2005). SERK and APOSTART. Candidate genes for apomixis in Poa pratensis. Plant Physiol. 138: 2185-2199.

Albrecht C, Russinova E, Hecht V, Baaijens E, et al. (2005). The Arabidopsis thaliana SOMATIC EMBRYOGENESIS RECEPTOR-LIKE KINASES1 and 2 control male sporogenesis. Plant Cell 17: 3337-3349.

Baudino S, Hansen S, Brettschneider R, Hecht VF, et al. (2001). Molecular characterisation of two novel maize LRR receptorlike kinases, which belong to the SERK gene family. Planta 213: 1-10.

Colcombet J, Boisson-Dernier A, Ros-Palau R, Vera CE, et al. (2005). Arabidopsis SOMATIC EMBRYOGENESIS RECEPTOR KINASES1 and 2 are essential for tapetum development and microspore maturation. Plant Cell 17: 3350-3361.

Cueva A, Concia L and Cella R (2012). Molecular characterization of a Cyrtochilum loxense Somatic Embryogenesis Receptorlike Kinase (SERK) gene expressed during somatic embryogenesis. Plant Cell Rep. 31: 1129-1139.

Dai HY, Li WR, Liu JH, Liu YX, et al. (2011). Agrobacterium-mediated transformation of Malus hupehensis var. pingyiensis using cotyledon explants. J. Shen. Agric. Univ. 6: 007.

Ge XX, Fan GE, Chai LJ and Guo WW (2010). Cloning, molecular characterization and expression analysis of a SOMATIC EMBRYOGENESIS RECEPTOR-LIKE KINASE GENE (CitSERK1-like) in Valencia sweet orange. Acta Physiol. Plant. 32: 1197-1207.

Gou X, He K, Yang H, Yuan T, et al. (2010). Genome-wide cloning and sequence analysis of leucine-rich repeat receptor-like protein kinase genes in Arabidopsis thaliana. BMC Genomics 11: 19.

Hecht V, Vielle-Calzada JP, Hartog MV, Schmidt ED, et al. (2001). The Arabidopsis SOMATIC EMBRYOGENESIS RECEPTOR KINASE 1 gene is expressed in developing ovules and embryos and enhances embryogenic competence in culture. Plant Physiol. 127: 803-816.

$\mathrm{Hu} H$, Xiong $L$ and Yang $Y$ (2005). Rice SERK1 gene positively regulates somatic embryogenesis of cultured cell and host defense response against fungal infection. Planta 222: 107-117.

Huang X, Lu XY, Zhao JT, Chen JK, et al. (2010). MaSERK1 gene expression associated with somatic embryogenic competence and disease resistance response in banana (Musa spp.). Plant Mol. Biol. Rep. 28: 309-316.

Ito Y, Takaya K and Kurata N (2005). Expression of SERK family receptor-like protein kinase genes in rice. Biochim. Biophys. Acta-Gene Struct. Express. 1730: 253-258.

Khan IA, Awan FS, Ahmad A and Khan AA (2004). A modified mini-prep method for economical and rapid extraction of genomic DNA in plants. Plant Mol. Biol. Rep. 22: 89.

Larkin MA, Blackshields G, Brown NP, Chenna R, et al. (2007). Clustal W and clustal X version 2.0. Bioinformatics 23: 2947-2948.

Liu H, Pu F, Ren Q and Liu L (1989). Some characters of apomictic Malus. Acta Hort. Sin. 16: 1-4. 
Livak KJ and Schmittgen TD (2001). Analysis of relative gene expression data using real-time quantitative PCR and the 2(-Delta Delta C(T)) method. Methods 25: 402-408.

Nolan KE, Irwanto RR and Rose RJ (2003). Auxin up-regulates MtSERK1 expression in both Medicago truncatula root-forming and embryogenic cultures. Plant Physiol. 133: 218-230.

Podio M, Felitti SA, Siena LA, Delgado L, et al. (2014). Characterization and expression analysis of SOMATIC EMBRYOGENESIS RECEPTOR KINASE (SERK) genes in sexual and apomictic Paspalum notatum. Plant. Mol. Biol. 84: 479-495.

Rodriguez-Leal D and Vielle-Calzada JP (2012). Regulation of apomixis: Learning from sexual experience. Curr. Opin. Plant Biol. 15: 549-555.

Sailer C, Schmid B, Stöcklin J and Grossniklaus U (2014). Sexual Hieracium pilosella plants are better inter-specific, while apomictic plants are better intra-specific competitors. Perspect. Plant Ecol. Evol. Syst. 16: 43-51.

Schellenbaum P, Jacques A, Maillot P, Bertsch C, et al. (2008). Characterization of VvSERK1, VvSERK2, VvSERK3 and VvL1L genes and their expression during somatic embryogenesis of grapevine (Vitis vinifera L.). Plant Cell Rep. 27: 1799-1809.

Schmidt ED, Guzzo F, Toonen MA and de Vries SC (1997). A leucine-rich repeat containing receptor-like kinase marks somatic plant cells competent to form embryos. Development 124: 2049-2062.

Sharma SK, Millam S, Hein I and Bryan GJ (2008). Cloning and molecular characterisation of a potato SERK gene transcriptionally induced during initiation of somatic embryogenesis. Planta 228: 319-330.

Shi YL, Guo SD, Zhang R, Meng ZG, et al. (2014). The role of somatic embryogenesis receptor-like kinase 1 in controlling pollen production of the Gossypium anther. Mol. Biol. Rep. 41: 411-422.

Shi YL, Zhang R, Lin Q and Guo SD (2012). Biological function of the somatic embryogenesis receptor-like kinases in plant. Yi Chuan 34: 551-559.

Shiu SH and Bleecker AB (2001). Receptor-like kinases from Arabidopsis form a monophyletic gene family related to animal receptor kinases. Proc. Natl. Acad. Sci. U.S.A. 98: 10763-10768.

Singla B, Tyagi AK, Khurana JP and Khurana P (2007). Analysis of expression profile of selected genes expressed during auxin-induced somatic embryogenesis in leaf base system of wheat (Triticum aestivum) and their possible interactions. Plant. Mol. Biol. 65: 677-692.

Somleva MN, Schmidt EDL and de Vries SC (2000). Embryogenic cells in Dactylis glomerata L.(Poaceae) explants identified by cell tracking and by SERK expression. Plant Cell Rep. 19: 718-726.

Spillane C, Steimer A and Grossniklaus U (2001). Apomixis in agriculture: The quest for clonal seeds. Sex. Plant. Reprod. 14: 179-187.

Tamura K, Dudley J, Nei M and Kumar S (2007). MEGA4: Molecular Evolutionary Genetics Analysis (MEGA) software version 4.0. Mol. Biol. Evol. 24: 1596-1599.

Thomas C, Meyer D, Himber C and Steinmetz A (2004). Spatial expression of a sunflower SERK gene during induction of somatic embryogenesis and shoot organogenesis. Plant Physiol. Biochem. 42: 35-42.

Thompson JD, Higgins DG and Gibson TJ (1994). Clustal W: Improving the sensitivity of progressive multiple sequence alignment through sequence weighting, position-specific gap penalties and weight matrix choice. Nucleic Acids Res. 22: 4673-4680.

Walker JC (1994). Structure and function of the receptor-like protein kinases of higher plants. Plant. Mol. Biol. 26: 1599-1609.

Wrzaczek M, Brosché M, Salojärvi J, Kangasjärvi S, et al. (2010). Transcriptional regulation of the CRK/DUF26 group of receptor-like protein kinases by ozone and plant hormones in Arabidopsis. BMC Plant Biol. 10: 95.

Zakizadeh H, Stummann BM, Lütken H and Müller R (2010). Isolation and characterization of four somatic embryogenesis receptor-like kinase (RhSERK) genes from miniature potted rose (Rosa hybrida cv. Linda). Plant Cell Tissue Organ Cult. 101: 331-338. 